\title{
NEW NARROW-BEAM NEUTRON SPECTROMETER IN COMPLEX MONITORING SYSTEM
}

\author{
E.A. Mikhalko \\ Polar Geophysical Institute, \\ Apatity, Russia,mikhalko@pgia.ru \\ Yu.V. Balabin \\ Polar Geophysical Institute, \\ Apatity, Russia, balabin@pgia.ru
}

\author{
E.A. Maurchev \\ Polar Geophysical Institute, \\ Apatity, Russia,maurchev1987@gmail.com \\ A.V. Germanenko \\ Polar Geophysical Institute, \\ Apatity, Russia,germanenko@pgia.ru
}

\begin{abstract}
In the interaction of cosmic rays (CRs) with Earth's atmosphere, neutrons are formed in a wide range of energies: from thermal $(E \approx 0.025 \mathrm{eV})$ to ultrarelativistic $(E>1 \mathrm{GeV})$. To detect and study CRs, Polar Geophysical Institute (PGI) uses a complex monitoring system containing detectors of various configurations. The standard neutron monitor (NM) 18-NM-64 is sensitive to neutrons with energies $E>50 \mathrm{MeV}$. The lead-free section of the neutron monitor (BSRM) detects neutrons with energies $E \approx(0.1 \div 1) \mathrm{MeV}$. Also, for sharing with standard detectors, the Apatity NM station has developed and installed a neutron spectrometer with three energy channels and a particle reception angle of
\end{abstract}

15 degrees. The configuration of the device makes it possible to study the degree of anisotropy of the particle flux from different directions. We have obtained characteristics of the detector (response function and particle reception angle), as well as geometric dimensions through numerical simulation using the GEANT4 toolkit [Agostinelli et al., 2003]. During operation of the device, we collected database of observations and received preliminary results.

Keywords: cosmic rays, nuclear physics, Monte Carlo method, particle detection.

\section{INTRODUCTION}

Cosmic rays (CR) are fluxes of charged particles of different energies, containing $~ 90 \%$ high-energy protons. Sources of galactic CRs (GCRs) are in interstellar space; GCRs come to Earth nearly isotropically. Solar CRs (SCRs) also consist largely of protons, but their source is the Sun. Penetrating into Earth's atmosphere, CRs interact with it in various ways, depending on energy of particles and density of ambient matter. In the upper layers, the atmospheric matter is discharged and charged particles lose their energy mainly during ionization. Starting at an altitude 15-20 km above sea level, the probability of nuclear reactions increases, producing cascades of secondary particles containing electromagnetic, hadronic, including neutron, components. Neutrons arising from these reactions have a wide range of energies (up to tens of $\mathrm{GeV}$ ) and propagate deep into the atmosphere reaching Earth's surface. Being distributed, they lose energy. Thus, Earth has neutron fluxes of various energies, beginning with thermal $(0.025 \mathrm{eV})$. However, low-energy neutron fluxes remain largely unaddressed, though they are involved in nuclear reactions.

In PGI, the flux of neutrons of various energies is monitored with a monitoring system [Maurchev, Balabin, 2016; Germanenko et al., 2016]. It includes a standard NM (SNM-15, 18-NM-64 counters, >50 MeV energy), lead-free NM section (LFNM, 4 SNM-15; the effective detection energy is from thermal energy to units of $\mathrm{MeV}$ ). To increase capabilities of the data acquisition system, in addition to the existing equipment we installed a narrow-beam neutron spectrometer (NBNS). A characteristic feature of this device is a ra- ther narrow beam $\left(\sim 15^{\circ}\right)$, which is determined by the configuration of the protection around the counters. The NBNS comprises three counters separated by moderator layers; hence there are three energy ranges. For more detail see Section 1. It should be noted that this construction has been calculated with the GEANT4 toolkit. The model is demonstrated in Figure 1.

\section{NARROW-BEAM NEUTRON SPECTROMETER}

The simulation has yielded geometric parameters of the device the front projection of which is shown in Figure 2. Helium counters SNM-18 are used as neutron detectors with an $\sim 70 \%$ detection efficiency. With increasing neutron energy, the counter efficiency decreases sharply, high-energy neutrons pass through the counter undetected. The counters are mounted above each other and are surrounded with a layer of paraffin $15 \mathrm{~cm}$ thick, which effectively slows down neutrons with an energy $E<1 \mathrm{MeV}$. Between the counters and the paraffin mass there is a layer of substance $4 \mathrm{~cm}$ thick, which contains boron that absorbs thermal neutrons. As a result, this detector is practically inaccessible to thermal neutrons coming into it at large angles and has a small detection angle of $15^{\circ}$. Between the three counters there is an additional layer of paraffin $5 \mathrm{~cm}$ thick, which slows down the neutrons coming into NBNS through the window. The upper counter, which is not closed with the paraffin layer, detects neutrons of thermal energies; the middle counter, separated by a $5 \mathrm{~cm}$ paraffin layer with an input window, neutrons with energies up to $E<100 \mathrm{keV}$. The lower counter, separated by a $10 \mathrm{~cm}$ 


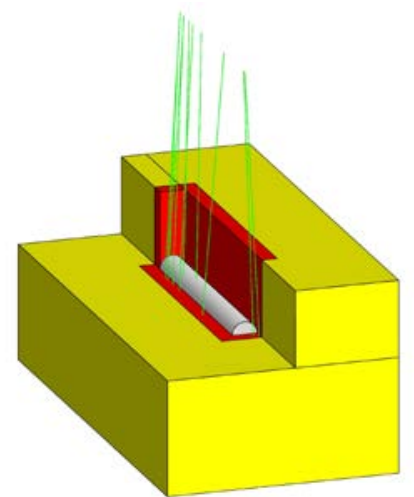

Figure 1. Three-dimensional model of NBNS, obtained from calculations with GEANT4. Yellow color indicates paraffin; red, the absorber region providing protection against thermal neutrons entering from the paraffin moderator. The upper counter is shown in gray; green lines are tracks of neutrons entering the inlet of NBNS

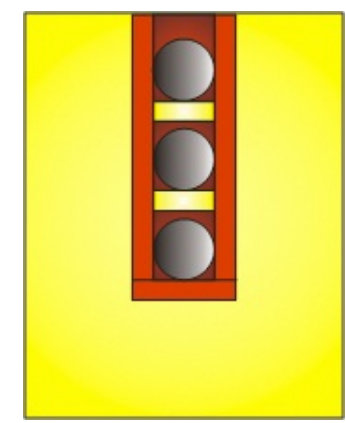

Figure 2. Cross-section of the projection of the NBNS model. The yellow color indicates paraffin acting as neutron moderator; red color, boron-containing absorber providing protection against thermal neutrons outside the receiving window; gray color, helium counters

paraffin layer with an input window, detects neutrons in an energy range $0.1<E<1 \mathrm{MeV}$. Among advantages of this detector is its mobility due to the use of SNM-18 with small dimensions (the length is $320 \mathrm{~mm}$, the diameter is $32 \mathrm{~mm}$ ). Owing to the use of these counters, NBNS does not have high statistical accuracy (its counting error is $1.9 \%$ for the upper counter, $3.2 \%$ for the middle one, and $4.7 \%$ for the lower one). The counting rate for each of the counters is very low, so we use daily means to observe neutron flux variations.

As detecting objects we use proportional ${ }^{3} \mathrm{He}$ counters; the effective cross-section for neutron capture at $E \approx 0.025 \mathrm{eV}$ is $\sigma=5400$ barn. The reaction channel can be expressed by the formula

$$
{ }^{3} \mathrm{He}_{2}+\mathrm{n} \rightarrow{ }^{3} \mathrm{H}_{1}+\mathrm{p}+0.765 \mathrm{MeV} \text {. }
$$

This reaction produces a tritium nucleus and proton, which cause secondary ionization. The reaction products, by ionizing the gas and accumulating on anode and cathode of the detector, form an electric pulse whose amplitude is proportional to the energy of this particle, or, more precisely, to the energy the particle expends on primary ionization of the detector medium [Shirokov, Yudin, 1980]. If the primary neutron has an energy exceeding the thermal one $(0.025 \mathrm{eV}<E<1 \mathrm{MeV})$, it slows down due to the reaction of elastic scattering by hydrogen atoms included in the paraffin. With one such inter- action the neutron loses up to half its energy. When $E>1$ $\mathrm{MeV}$, the cross section of the scattering reaction decreases sharply and the neutron is most likely to fly through the detector material.

\section{CALCULATION OF NBNS DETECTION EFFICIENCY}

To calculate the geometric parameters, which determine the viewing angle, and the response function of the device as a function of energy and particle arrival angle, we employ the GEANT4 toolkit. In the simulation, we specify the calculation of material parameters and the configuration of the receiving window of the device in such a way as to maximize the range of measured energies as well as to provide the narrow beam of the detector. The cross-sections of neutron interactions with matter up to $E=10 \mathrm{MeV}$ have been selected using the ENDF/B-VII.1 database [Chadwick et al., 2011]. We also use standard electromagnetic processes to simulate the operation of the ${ }^{3} \mathrm{He}$ counters.

The simulated experiment has two stages. At the first stage, the upper counter is placed under the moderator plate with different thicknesses $(2,4,8 \mathrm{~cm})$. The particle generator is implemented so that the neutrons fall strictly perpendicular, having a uniform distribution over the window area. At the second stage of the experiment, the plate is removed and the direction of particles is generated at different angles $\left(0,15\right.$ and $\left.45^{\circ}\right)$ with respect to the central part of NBNS. The detection efficiencies obtained from these calculations are shown in Figure $3, a, b$. It can be seen that the presence of the plate between the counters makes it possible to realize the calorimeter function in the device, and the window provides the narrow beam of the detector, excluding neutrons entering at large angles.

\section{ANNUAL VARIATIONS OF NEUTRON FLUXES OF DIFFERENT ENERGIES}

Data were acquired from NBNS for a year from the date of its implementation (from January 1, 2016 to December 31, 2016); findings were stored in the database of the general monitoring system. Counting rates in NBNS together with NM and LFNM depending on days of year are shown in Figure 4. It can be seen (Figure 4 $a, c)$ that readings of the NBNS upper counter agree well with neutron detection results obtained with LFNM and indicate the presence of seasonal variations in the intensity of neutrons with energies from $0.025 \mathrm{eV}$ to 1 $\mathrm{MeV}$. There is no seasonal dependence in the counting rate for the standard NM 18-NM-64, as for the NBNS lower counter (Figure $4, d, e$ ). This is attributed to the sensitivity to more energetic neutrons. The NBNS middle counter has a weak seasonal variation (Figure 4, b).

\section{CONCLUSION}

The narrow-beam thermal neutron detector significantly increases the capabilities of the complex data acquisition system installed at the NM station. This device 

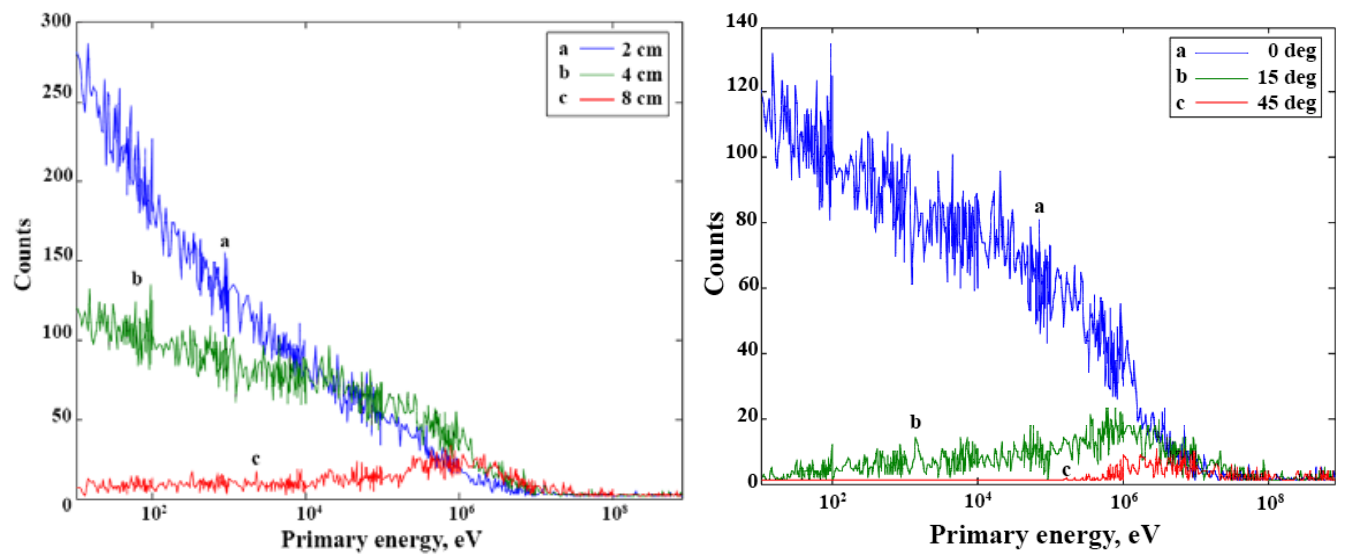

Figure 3. Calculated neutron detection efficiency dependng on the plate thickness above the counter $(a)$ and on the particle arrival angle $(b)$
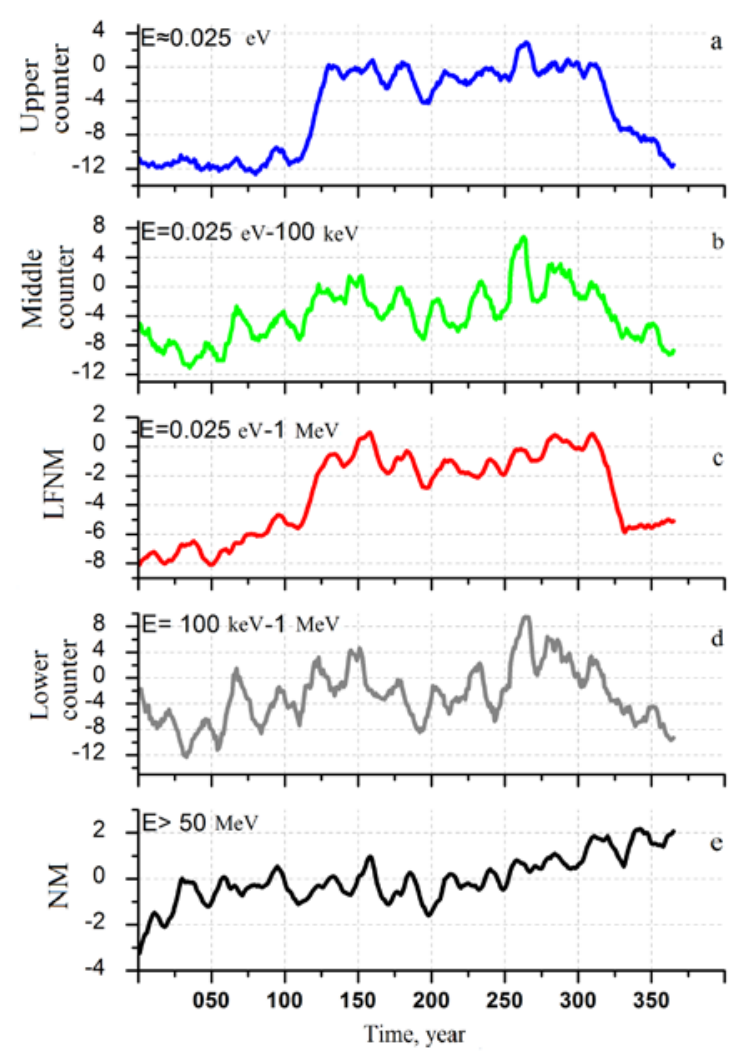

Figure 4. Annual dynamics in detector measurements: upper counter of the neutron spectrometer $(a)$; middle counter of the spectrometer $(b)$; lead-free section of the neutron monitor $(c)$; lower counter of the spectrometer $(d)$; standard neutron monitor (e)

has been shown not only to have spectrometer functions, including three detection channels, but also to allow measurements of the degree of thermal neutron flux anisotropy in the surface layer. With NBNS we carried out observations over a year and acquired data that confirm the complete correspondence of the actual and calculated efficiency of the device.

It should be noted that the device can not only operate as part of the existing data acquisition system, but can also supplement knowledge of the thermal part of near-Earth neutron spectrum obtained in [Pioch et al., 2011].

\section{REFERENCES}

Agostinelli S., Allison J., Amako K., Apostolakis J., Araujo H., Arce P., Asai M., Axen D., Banerjee S., Barrand G., Behner F., Bellagamba L., Boudreau J., Broglia L., Brunengo A., Burkhardt H., Chauvie S., Chuma J., Chytracek R., Cooperman G., Cosmo G., Degtyarenko P., Dell'Acqua A., Depaola G., Dietrich D., Enami R., Feliciello A., Ferguson C., Fesefeldt H., Folger G., Foppiano F., Forti A., Garelli S., Giani S., Giannitrapani R., Gibin D., Gomez Cadenas J.J., Gonzalez I., Gracia Abril G., Greeniaus G., Greiner W., Grichine V., Grossheim A., Gumplinger P., Hamatsu R., Hashimoto K., Hasui H., Heikkinen A., Howard A., Ivanchenko V., Johnson A., Jones F.W., Kallenbach J., Kanaya N., Kawabata M., Kawabata Y., Kawaguti M., Kelner S., Kent P., Kodama T., Kokoulin R., Kossov M., Kurashige H., Lamanna E., Lampen T., Lara V., Lefebure V., Lei F., Liendl M., Lockman W., Longo F., Magni S., Maire M., Medernach E., Minamimoto K., Mora de Freitas P., Morita Y., Murakami K., Nagamatu M., Nartallo R., Nieminen P., Nishimura T., Ohtsubo K., Okamura M., O’Neale S., Oohata Y., Paech K., Perl J., Pfeiffer A., Pia M.G., Ranjard F., Rybin A., Sadilov S., Salvo E Di, Santin G., Sasaki T., Savvas N., Sawada Y., Scherer S., Sei S., Sirotenko V., Smith D., Starkov N., Stoecker H., Sulkimo J., Takahata M., Tanaka S., Tcherniaev E., Safai Tehrani E., Tropeano M., Truscott P., Uno H., Urban L., Urban P., Verderi M., Walkden A., Wander W., Weber H., Wellisch J.P., Wenaus T., Williams D.C., Wright D., Yamada T., Yoshida H., Zschiesche D. Geant 4 - a simulation toolkit. Nuclear Instruments and Methods in Physics Research. Section A: Accelerators, Spectrometers, Detectors and Associated Equipment. 2003, vol. 506, no. 3, p. 250. DOI: 10.1016/S0168-9002(03)01368-8.

Chadwick M.B., Herman M., Obložinský P., Dunn M.E., Danon Y., Kahler A.C., Smith D.L., Pritychenko B., Arbanas G., Arcilla R., Brewer R., Brown D.A., Capote R., Carlson A.D., Cho Y.S., Derrien H., Guber K., Hale G.M., Hoblit S., Holloway S., Johnson T.D., Kawano T., Kiedrowski B.C., Kim H., Kunieda S., Larson N.M., Leal L., Lestone J.P., Little R.C., McCutchan E.A., MacFarlane R.E., MacInnes M., Mattoon C.M., McKnight R.D., Mughabghab S.F., Nobre G.P.A., Palmiotti G., Palumbo A., Pigni M.T., Pronyaev V.G., Sayer R.O., Sonzogni A.A., Summers N.C., Talou P., Thompson I.J., Trkov A., Vogt R.L., van der Marck S.C., Wallner A., White M.C., Wiarda D., Young P.G. ENDF/B-VII.1 Nuclear data for science and technology: cross sections, covariances, fission product yields and decay data. Nuclear Data Sheets. 2011, vol. 112, iss. 12. pp. 2887-2996. DOI: 10.1016/j.nds. 2011.11.002. 
Germanenko A.V., Balabin Yu.V., Gvozdevsky B.B., Shchur L.I. Nature of variations of gamma-radiation during precipitation. Solnechno-zemnaya fizika [Solar-Terrestrial Physics]. 2016, vol. 2, no. 1. pp. 56-63. (In Russian). DOI: $10.12737 / 19880$.

Maurchev E.A., Balabin Yu.V. RUSCOSMIC — the new software toolbox for detailed analysis of cosmic ray interactions with matter. Solar-Terr. Phys. 2016, vol. 2, no. 4. pp. 3 10. DOI: $10.12737 / 24269$.

Pioch C., Mares V., Vashenyuk E.V., Balabin Yu., Rühm W. Measurement of cosmic ray neutrons with Bonner sphere spectrometer and neutron monitor at $79^{\circ} \mathrm{N} / /$ Nuclear Instruments and Methods in Physics Research. Section A. 2011, vol. 626, pp. 5157. DOI: 10.1016/j.nima.2010.10.030.
Shirokov Yu.M., Yudin N.P. Yadernaya fizika [Nuclear Physics]. $2^{\text {nd }}$ Edition. Moscow, Nauka Publ., 1980. 728 p. (In Russian).

How to cite this article

Mikhalko E.A., Balabin Yu.V., Maurchev E.A., Germanenko A.V. New Narrow-Beam Neutron Spectrometer in Complex Monitoring System. Solar-Terrestrial Physics. 2018, vol. 4, no. 1, pp. 71-74 . DOI: $10.12737 /$ stp-41201808. 